\title{
Die Ethopöie des Nikephoros Chrysoberges über Julians Rhetorenedikt.
}

Im Jahre 1892 veröffentlichte Maximilian Treu ${ }^{1}$ ) aus dem Codex Vindobonensis Gr. 321 drei Reden eines bisher noch nicht bekannten Nikephoros Chrysoberges und versah diese Anekdota mit einem gründlichen literarischen, biographischen und exegetischen Kommentar. Er teilte hierin u. a. mit, daB dieser Byzantiner, der im 12. Jahrhundert in Konstantinopel als Kleriker und Lehrer der Rhetorik tätig war, in der genannten Handschrift, von andereu Werken abgesehen, noch durch neun $\pi \varrho \circ \gamma v \mu \nu \alpha \dot{\sigma} \sigma \alpha \tau \alpha$ vertreten ist. Das siebente in der Reihe (fol. 258r) ist überschrieben:

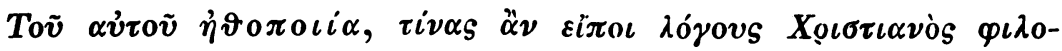

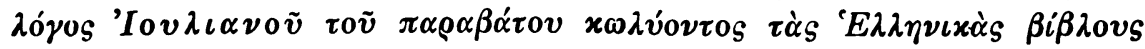

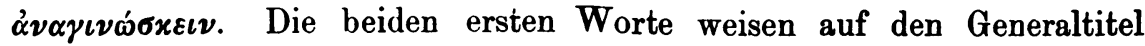
der Progymnasmata zurück, wo der Name und der Stand des Ver-

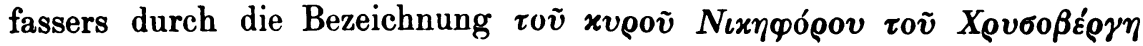

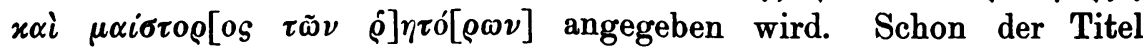
unserer Ethopöie sticht von der gewohnten Gegenstandslosigkeit und Verschwommenheit ähnlicher byzantinischer Elaborate inhaltlich und formell durch eine gewisse prägnante Bestimmtheit so vorteilhaft ab, daB nicht nur Krumbacher in seiner byzantinischen Literaturgeschichte ${ }^{2}$ ), sondern auch neuere Julianforscher, wie Bidez und Cumont ${ }^{3}$ ), ihrer besonders Erwähnung tun zu müssen glaubten, obschon ihr Inhalt ihnen noch nicht bekannt war. Es ist ja auch in der Tat schon rein an und für sich ein interessantes Faktum, daB ein von Julian gleich im Anfang seiner Regierung erlassenes Kulturkampfedikt noch ums Jahr 1200 als rhetorischer Übungsstoff Verwendung findet. $\mathrm{DaB}$ freilich gerade unser Byzantiner auf ihn verfiel, erklärt sich sehr einfach aus

1) Nicephori Chrysobergae ad Angelos orationes tres. Wissensch. Abhandlung z. Progr. des K. Friedrichs-Gymn. z. Breslau.

2) 2. Aufl. S. 470 .

3) Recherches sur la tradition manuscrite des lettres de l'empereur Julien. Bruxelles 1898 p. $26 \mathrm{n}$. 
seinem geistlichen Rhetorenamite, demgemäß er neben der bereits angegebenen Standesbezeichnung :auch noch das im Spezialtitel der fünften Rede mitgeteilte Ehrenprädikat eines $\delta \iota \delta \alpha^{\prime} \sigma x \alpha \lambda o_{S} \lambda o \gamma \iota \omega^{\prime} \tau \alpha \tau o s$ führte. Da wir uns schon seit Jahren mitt der literarischen Hinterlassenschaft des Apostaten im allgemeinen und mit ihren Nachklängen in der späteren Polemik im besonderen beschhäftigen, wandten wir uns um näheren AufschluB an den Entdecker der Ethopöie und erhielten von ihm eine sorgfältige Abschrift ihrres Textes zur Verfügung gestellt. Wir möchten nicht verfehlen, ihm auch hier noch einmal für seine uneigennützige Liebenswürdigkeiit verbindlichst zu danken. Der Text ist folgender:

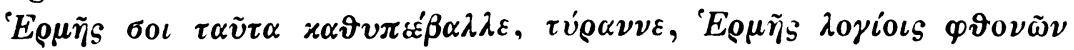

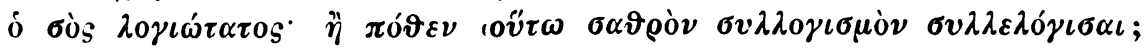

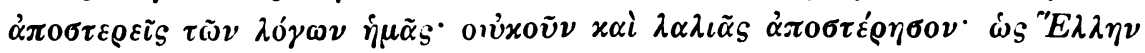

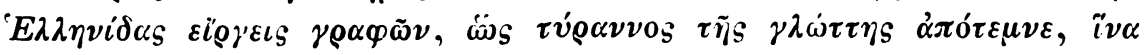

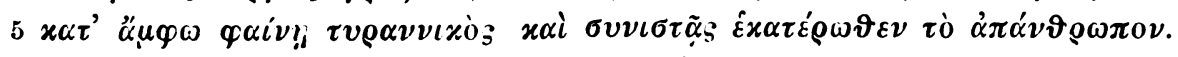

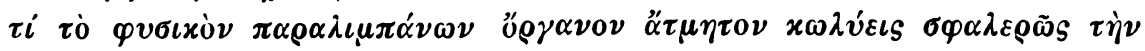

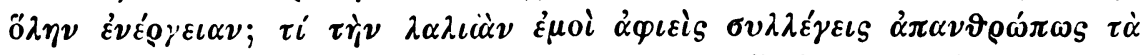

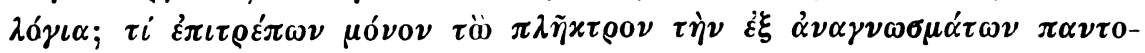

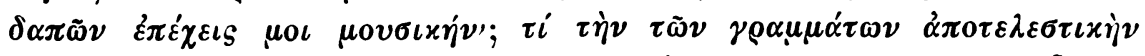

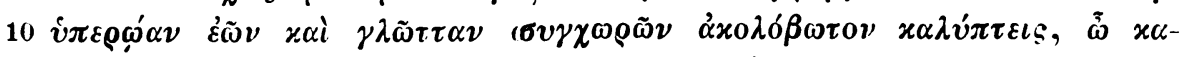

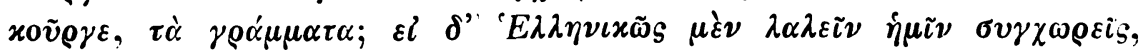

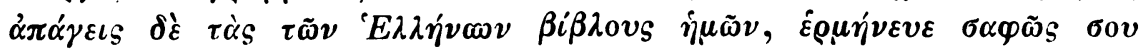

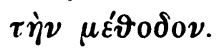

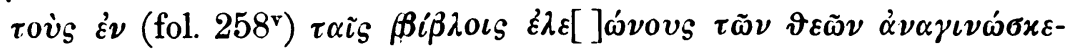

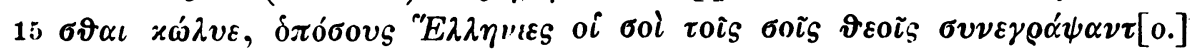

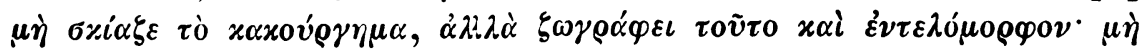

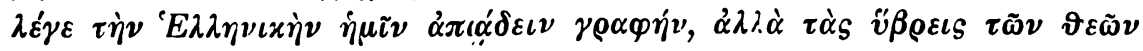

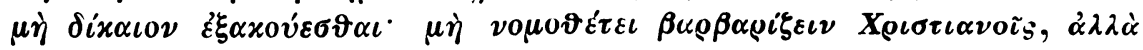

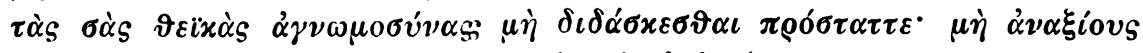

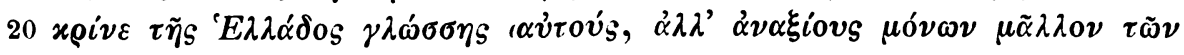
$\mu v \sigma \tau \iota x \tilde{\omega} \nu$ боv $x \alpha \tau \alpha x \varrho \circ \alpha \dot{\sigma \varepsilon \omega \omega \nu}$.

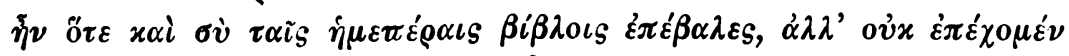

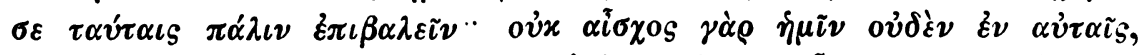

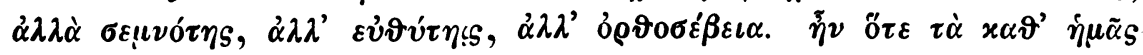

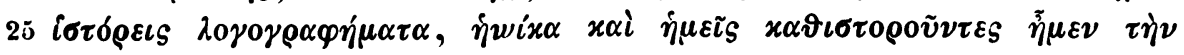

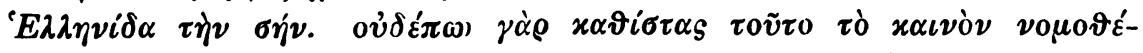

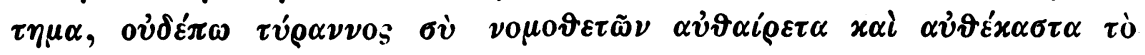

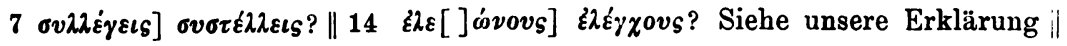

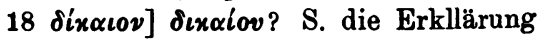




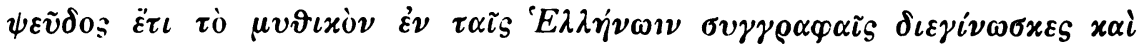

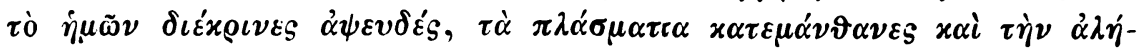

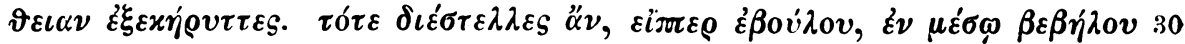

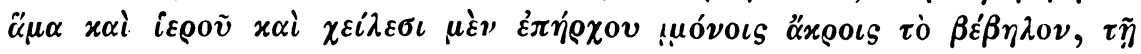

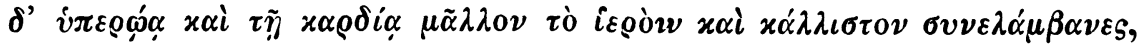

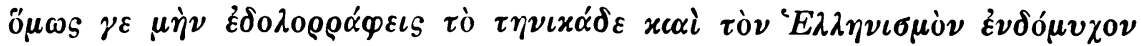

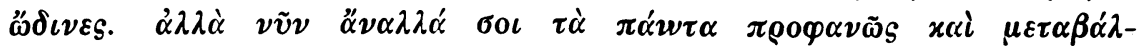

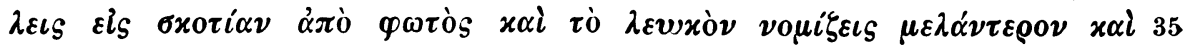

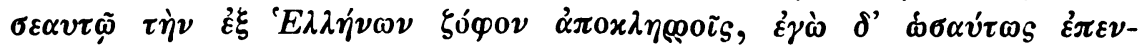

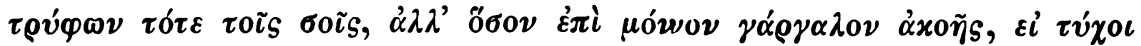

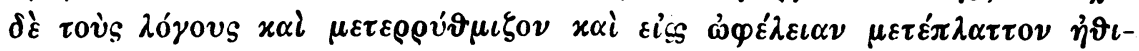

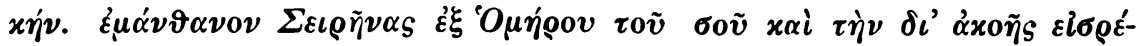

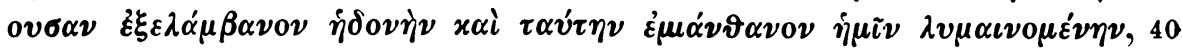

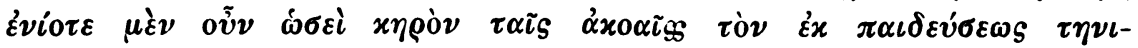

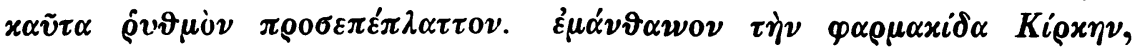

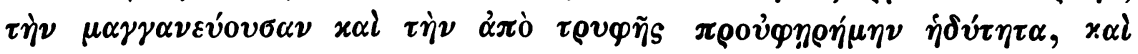

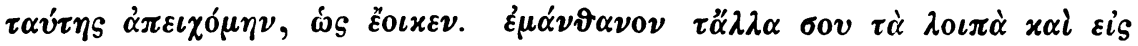

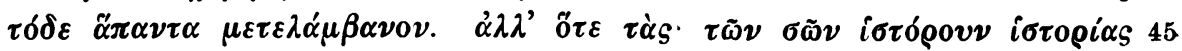

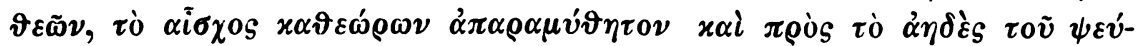
dovs żixa\}ov.

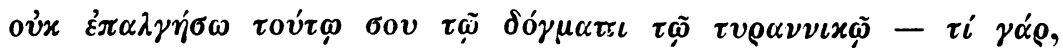

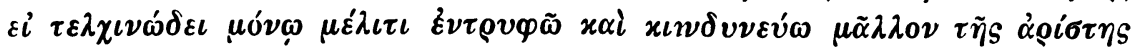

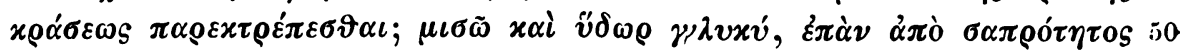

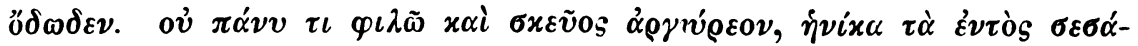

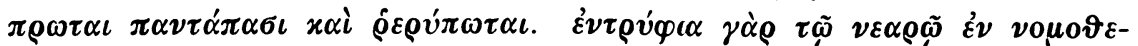

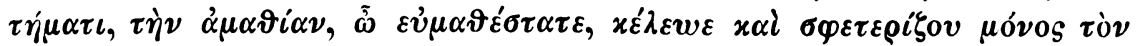

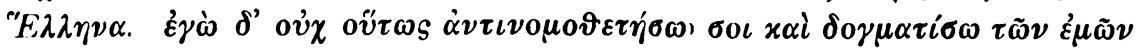

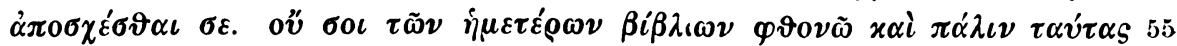

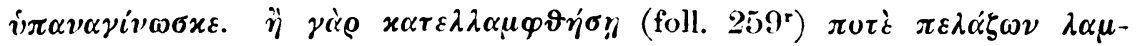

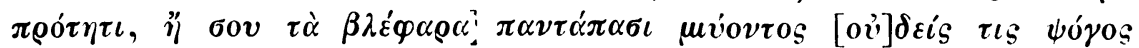
$\ddot{\varepsilon} \sigma \varepsilon \tau \alpha \iota \pi \rho \dot{g} \tau \dot{\partial} \nu \ddot{\eta} \lambda \iota \iota \nu$.

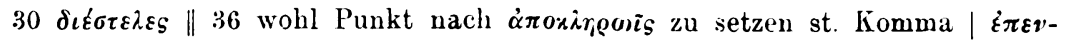

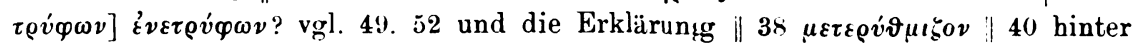

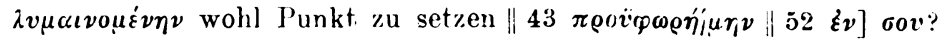

Wir geben die handschriftliche Fassumg, abgesehen ron einigen selbstverständlichen Verbesserungen, getreu wieder, nur daß wir den im Original fortlaufend geschriebenen Wortlaıt dem Inhalt entsprechend in vier Abschnitte teilen. Über die Art, wie einige Textverderbnisse geheilt werden könnten, gibt unsere kieine Acinotatio Aufschlub, ohne dab sie jedoch einen Anspruch auf Vollstï̈ndigkeit erheben möchte 
Namentlich in syntaktischen Fragen halten wir einen konservativ reservierten Standpunkt für empfehlenswerter als irgendwelche mehr oder minder willkürlichen Änderungen.

Beherzigt man, daB unsere Ethopöie als solche eine bloß fingierte Entgegnung ist und auch in Titel ausdrücklich dafür ausgegeben wird, und daB zwischen ihrem Gegenstand und ihrer Abfassung ein Zeitraum von mehr. als 800 Jahren liegt, so wird man ihr selbst bei der gröBten Voreingenommenheit gegen derartige Erzeugnisse der byzantinischen Rhetorik dennoch zweierlei nicht abstreiten dürfen: eine temperamentvolle Wärme des Tons, die an manche Lukian-, Julian- und Libaniosscholien erinnert, und eine pointierte Schärfe der Gedanken, die sich über das übliche Niveau der einseitig fanatischen Ablehnung erbebt. Weder ihr Ethos noch ihre Argumente können das geistige Eigentum des Nikephoros Chrysoberges sein. Dies ist schon von vornherein einleuchtend und braucht nicht erst durch die Kontrastierung mit seinen matteu und dürftigen Reden bewiesen werden Wer ist also der Gewährsmann, der aus ihr herausspricht? Der 'Titelfiktion entsprechend wohl ein Zeitgenosse des Kaisers Julian, der es diesem verübelt, daB er die Christen an der Lektüre der hellenistischen Bücher hindern will. Nun besitzen wir tatsächlich nur noch eine einzige christliche Kontroversschrift gegen Julians Rhetorenedikt, auf welche diese chronologische Bestimmung paßt, nämlich die erste Invektive des Gregorios von Nazianz, die dieser zusammen mit der zweiten nicht lange nach dem Tode des Apostaten verfaBte. Er verfolgte dabei, wie er selbst I c. 4 sagt, den Zweck, diesen $\dot{v} \pi \dot{\imath} \rho \tau \tilde{\eta} s$ cis $\lambda \dot{o} \gamma_{0} v_{S}$ $\pi \alpha \rho \alpha \nu o \mu i \alpha s$ zu züchtigen oder, wie sein Biograph Gregorios Presbyter

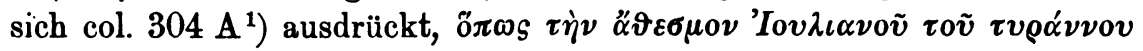

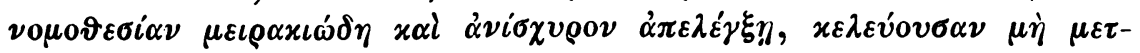

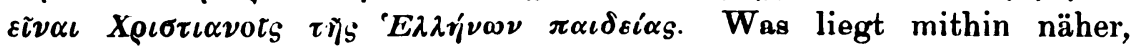
als in unserer Ethopöie ein Derivat aus dieser den Byzantinern schon an und für sich so nahe liegenden Quelle zu vermuten?

Beginnen wir unsere vergleichende Analyse mit dem Titel der Ethopöie, so verdienen hierin drei Besonderheiten hervorgehoben zu werden. Chrysoberges legt seine Entgegnungen einem christlichen

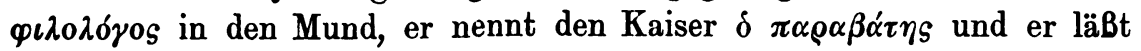
den Leser im unklaren, wen dieser an der hellenistischen Lektüre hindern will. Gerade diese Unklarheit, um mit dem letzten Punkte anzufangen, ist auch für Gregor charakteristisch: Auch er sagt in den

1) Wir zitieren ihn wie seinen Helden nach dem Migneschen Abdruck der Mauriner Ausgabe, Patrologiae curs. compl. Ser. Gr. t. 35. 
auf das Rhetorenedikt bezüglichen Teilen seiner Invektiven (I c. 4. 5 . 6. 96 ff., II c. 39) nicht deutlich, ob man es mit einem bloBen Lehroder auch mit einem Lernverbot zu tun habe. ${ }^{1}$ ) Ferner bezeichnet er

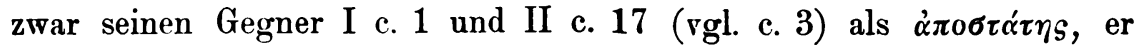
behauptet aber II c. 19 ausdrücklich, die einzige Empfehlung zur Erlangung von Amt und Würden sei bei ihm die $\pi \alpha \varrho \alpha \beta \beta \alpha \sigma \iota s$ gewesen.

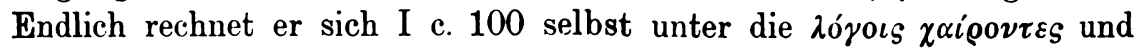
redet andrerseits I c. 105 den Kaiser ironisch $\check{\omega} \ldots \sigma v^{\prime} \ldots$. . $\iota \lambda o \lambda o ́ \gamma \varepsilon$ an. Hierzu kommt noch, daB er I c. 100 an jeden Literaturfreund die direkte Aufforderung richtet, seinen Groll zu teilen, worin man geradezu die Veranlassung zur Abfassung unserer Ethopöie erblicken könnte.

Gehen wir nun zu den Werken selbst über, so haben sie beide den Grundzug der Ethopöie miteinander gemein, den Aquila Romanus p. 24 mit den Worten: „certis quibusdam personis verba accommodate adfingimus vel ad improbitatem earum demonstrandam vel ad dignitatem" kennzeichnet; nur ist bei Gregor nicht wie bei Chrysoberges ein fremder "quidam", sondern er selbst die Person, die er in den bedeutendsten Partien ihre Apostrophen an den noch als lebend vorgestellten Gegner richten läßt, während er in den übrigen Abschnitten in der dritten Person von ihm spricht. Daher kommt es auch, daB gerade diese apostrophierenden Kapitel der Invektiven die meisten Parallelstellen zu unserer Ethopöie abgeben. Auch die stehenden Epitheta, die der Kaiser an beiden Orten erhält, sind dieselben. Wir stellen sie hier nebst einigen andern, auf sein Edikt bezïglichen, zusammen:

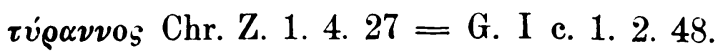

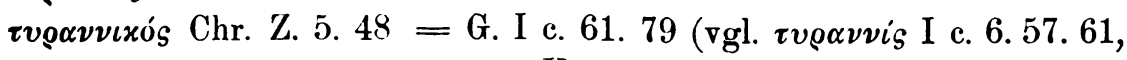
II c. 3 ; $v \varrho \varrho \nu \nu \varepsilon \varepsilon ́ \omega$ I c. 6 , II c. 21.37$)$.

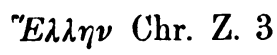
vgl. G. I c. $105 \hat{\dot{\omega}} \phi \iota \lambda \dot{\varepsilon} \lambda \lambda \eta \eta^{\prime} \sigma \dot{v}$.

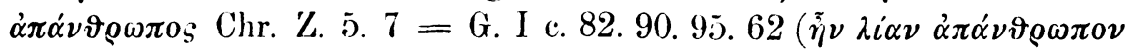

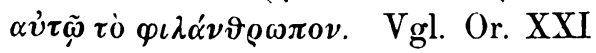
c. 32). Vgl. II c. 19. 24. - $\alpha \pi \alpha \nu \vartheta \rho \omega$. $\pi i \alpha$ I c. $91 ; \varphi \iota \lambda \alpha^{\prime} \nu \vartheta \rho \omega \pi \operatorname{s}^{\circ}$ iron. I c. 93 ,

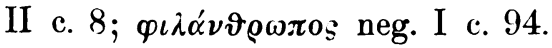

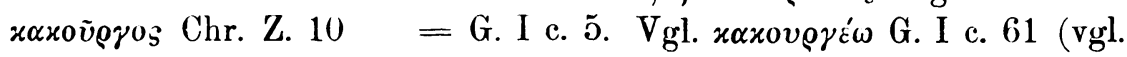

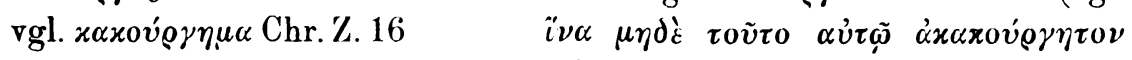
$\mu \varepsilon^{\prime} \nu !$ I c. 110) xaxov́ogrjuc II c. 14; xaxovoyia I c. 82.110.

1) Über diese Frage stellt das ältere Material gut zusan:mon Gothofiecus zu Cod. Theodos. XIII 3,5 t. V p. 35; das neuere verzeichnet Allard, Julien l'Apostat t. II p. 352 sq. 


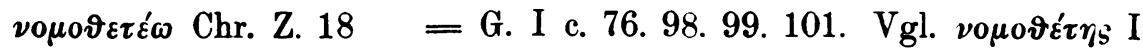

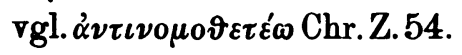

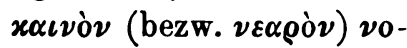

$\mu 0 \vartheta \varepsilon^{\prime} \eta \mu \alpha \mathrm{Chr}$. Z. 26.52

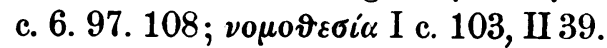

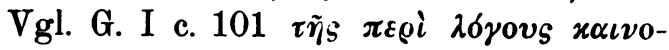

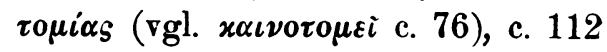

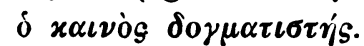

$\delta \delta \gamma \mu \alpha$ Chr. Z. 48

$=$ G. I c. 101 , II c. 39 .

$\delta \circ \gamma \mu \alpha \tau i \xi \omega$ Chr. Z. 54

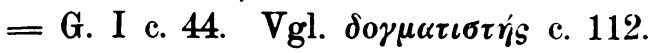

Weist schon diese Übereinstimmung auf die Benützung von Gregors Invektiven hin, so ist dies erst recht bei dem Gedankengang unserer Ethopöie der Fall. Zunächst bemängelt dieselbe die Folgerichtigkeit des kaiserlichen Ediktes und geht mit dem höhnisch-pathetischen Ein-

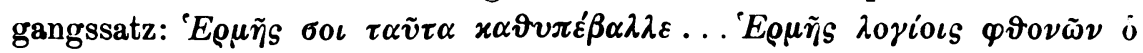

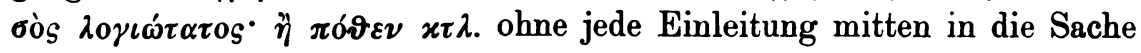
hinein. Hiemit vergleiche man die höhnische Frage Gregors I c. 101

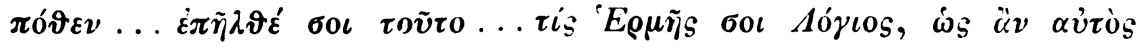

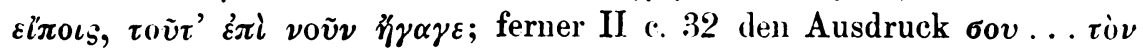
$\Lambda$ ócov und c. 4 den dem Kaiser selbst statt seinem Schutzgott ge-

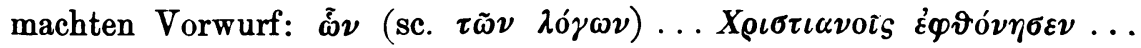

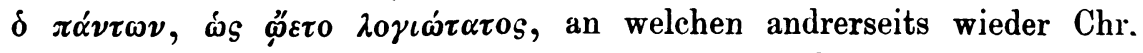

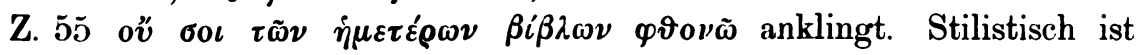
hierzu noch zu bemerken, daB der hämische Zusatz $\delta$ бòs zu dem Namen des Hermes einer echt gregorianischen Eigentümlichkeit ent-

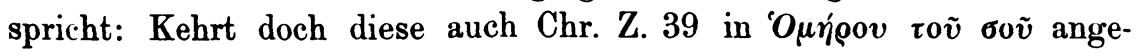

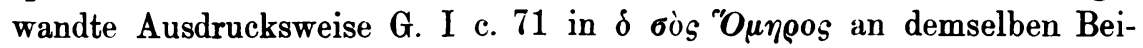
spiele wieder. Ebenso ist der häufige Gebrauch der Anaphora, wie sie gleich im ersten Satze auftritt, dem Nazianzener eigen. Vgl. u. a. I c. 1.2 .3 , II c. 32 . Wenn sodann Chr. Z. 2 der mangelhafte SchluB

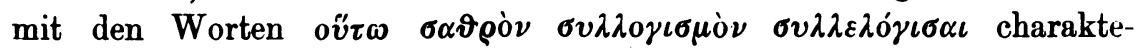

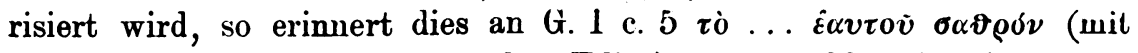

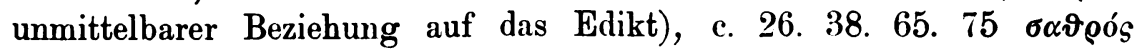
einerseits und an G. I c. $104 \dot{\alpha} \sigma v \lambda \lambda o_{0} \iota \sigma \tau o \nu$ (sc. das Edikt u. ä.; vgl.

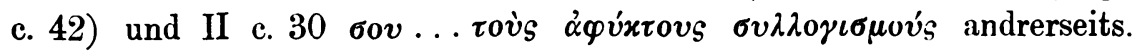
Aus Gregor I c. 104 erfahren wir, daB es sich dabei um den SchluB handelt, wenn zwei Dinge derselben Sphäre angehören könnten, müBten sie deshalb auch identisch sein, $d$. h. auf unsern Fall angewendet, wenn irgendwelche Leute sowohl der Sprache als auch der Religion nach Hellenisten sein könnten, müBten deshalb auch die hellenistische Literatur und die hellenistische Religion zusammenfallen. Nach dieser Logik würden, so meint der Nazianzener, die Christen als Gegner der hellenistischen Religion allerdings mit Fug und Recht von der hellenistischen Literatur ausgeschlossen. Chrysoberges teilt Z. 3 (vgl. Z. 7) 
nicht mehr als das faktische Ergebnis dieses Trugschlusses mit, indem

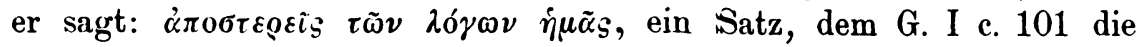

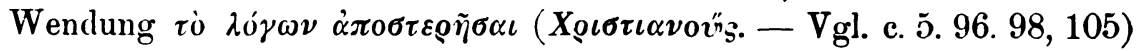
wörtlich und las c. 102 mitgeteilte Zitat aus dem Rhetorenedikt dem

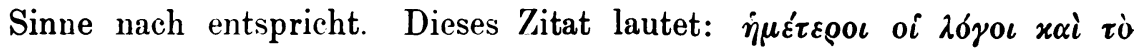

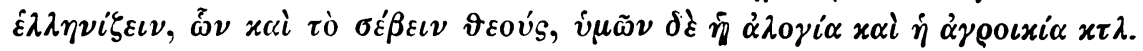
und besagt dasselbe, was die Ethopöie 7. 16-18 umständlicher zum Ausdruck bringt.

Der hier Z. 3 folgende Einwand, Julian hätte die Christen, statt ihnen bloB die hellenistische Literatur unzugänglich zu machen, lieber gleich des physischen Sprechvermögens berauben sollen, ist bei Gregor so wenig vorgebildet, wie die Z. 6-10 (vgl. Z. 29) dazu verwendete physiologische Weisheit. Doch betont der Kirchenvater I c. 5, die Christen würden, so lange sie noch im Besitz einer $\gamma \lambda \tilde{\omega} \sigma \sigma \alpha(=\mathrm{Chr}$. Z. 4. 10) seien, sich nicht von dem Bekennen ihres Glaubens abhalten lassen, ein Argument, woraus sich gleichzeitig auch der Sinn des Z. 6

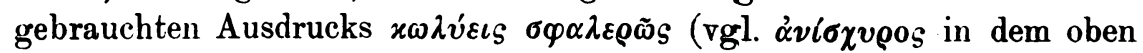
S. 128 zitierten Satz aus Gregorios Presbyter) ergibt; ferner vergleicht er in I c. 6 den Kaiser mit einem Athleten, der seinen Partner zwingt, mit verstümmelten Gliedern anzutreten; weiterhin erklärt er I c. 5 und c. 106 die Sprache für eine Funktion $\tau \tilde{y}_{\zeta \varsigma} \gamma \lambda \dot{\omega} \sigma \sigma \eta s$ bezw. der $\varphi \omega \nu \eta \tau \iota x \grave{~}$

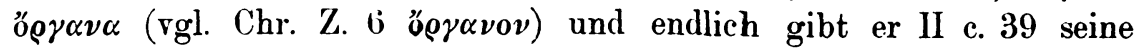
Genugtuung darüber zu erkennen, daß es nicht gelungen sei, die Christen zum Verstummen zu bringen, bezw. ihrer $\gamma \lambda \tilde{\omega} \sigma \sigma \alpha$ Fesseln an-

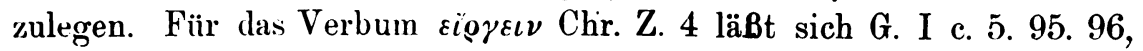
für $\varkappa \omega \lambda v^{\prime} \varepsilon \iota$ Chr. Z. 6. 15 (und im Titel): G. I c. 5 und für $\varepsilon \dot{\pi} \varepsilon \dot{\varepsilon} \varepsilon \iota \nu$ Chr. Z. 9 (=22): G. I c. 5. 6, II c. 39 als Parallele anführen. AuBer-

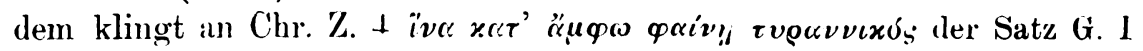

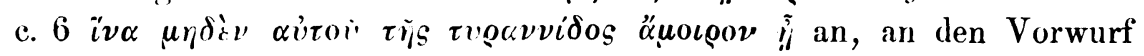
Chr. Z. 6, Julian rerkürze die rolle Redefreiheit, G. I c. 95.105 die

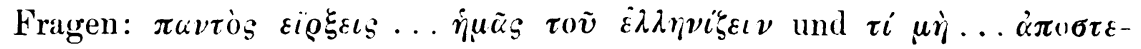

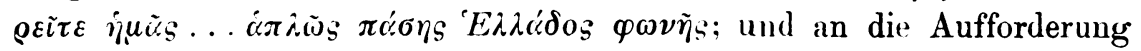
Chr. Z. 12, eine genaue Frklärung zu geben, G. I c. 101 die Mahnung:

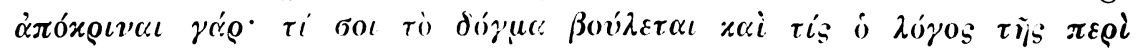

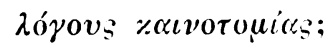

Im zweiten Abschnitt geht die Ethopïie zu einer Kritik der hellenistischen Theologir über und rät dem Kaiser, der Verbreitung der hellenistischen Literatur überhaupt zu steuern, anstatt sie blob den Christen vorzuenthalten Auch diese Gedank*n finden sich bereits bei Gregor, und zwar hauptsächlich in seiner dramatischen Satire auf den

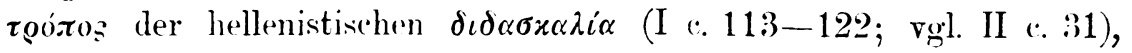


wie diese in den von Julian errichteten $\delta \iota \delta \alpha \sigma x \alpha \lambda \varepsilon i \alpha$ (I c. 111; vgl. c. $120.122=$ Chr. Z. $\left.19 \delta \iota \delta \alpha^{\prime} \sigma \kappa \omega\right)$, in Gestalt von $\alpha \nu \alpha \gamma \nu \omega ́ \sigma \varepsilon \iota$ (vgl.

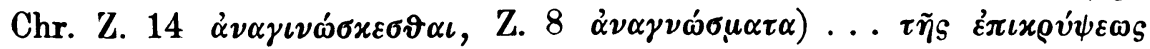

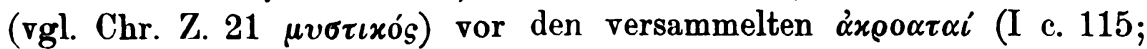

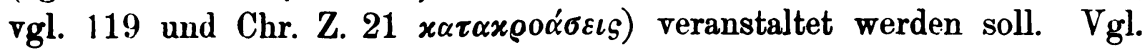

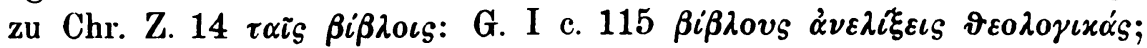

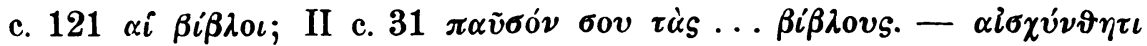

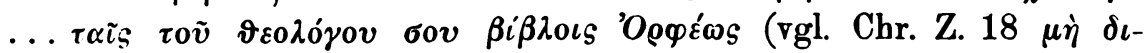

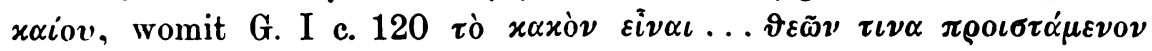

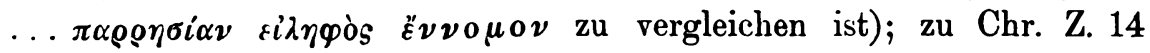

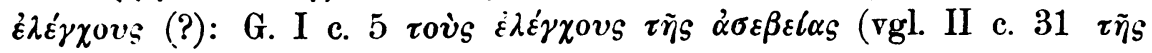

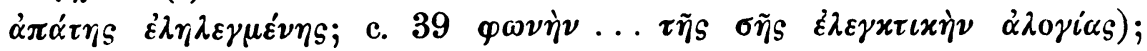

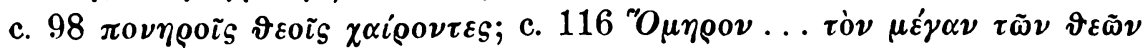

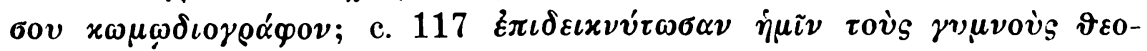

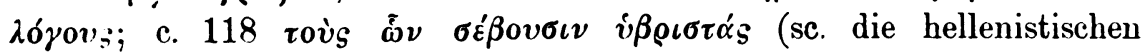

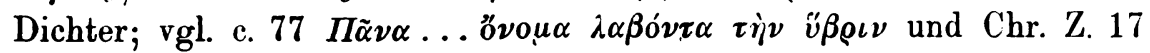

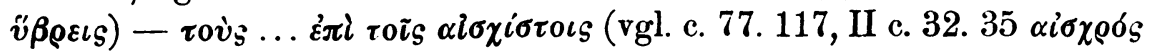

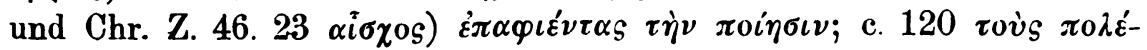

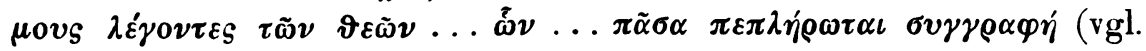

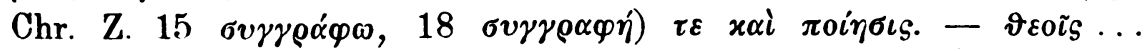

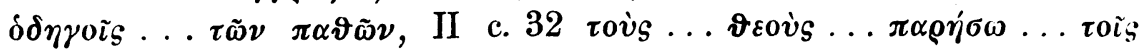

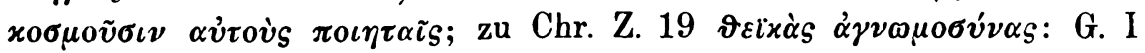

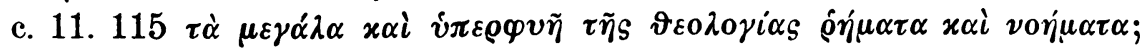
c. $116 \dot{\alpha} \sigma \chi \eta \mu о \nu \varepsilon \tilde{\nu} \nu$ (sc. heiBt Hera den Zeus), II c. $31 \tau \dot{\eta} \nu \dot{\alpha} \sigma \chi \eta \mu \sigma \sigma v ́ v \eta \nu$ (sc. der Götter; vgl. c. 22).

Viel deutlicher werden die Anklänge an Gregor wieder im dritten Abschnitt unserer Ethopöie. Aus diesem erkennen wir, daB der Sprecher nicht nur ein Zeitgenosso des Kaisers ist, sondern daB er gleich diesem einst Griechenland besuchte, da $B$ er ihn damals noch als Christen kannte, ihm aber gleichwohl nicht mehr trauen zu dürfen glaubte.

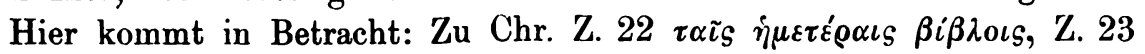

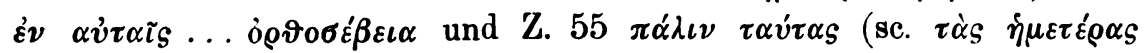

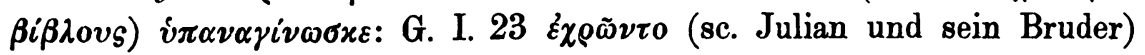

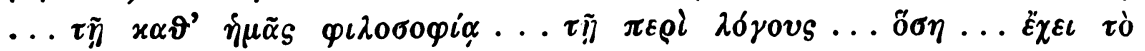

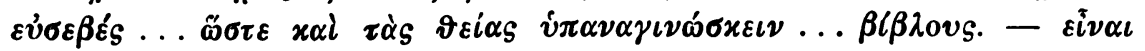

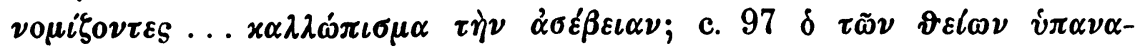

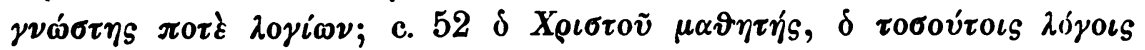

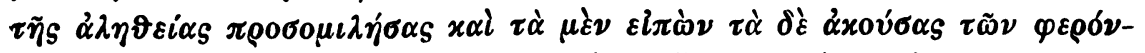

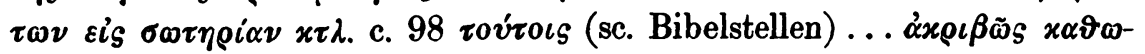

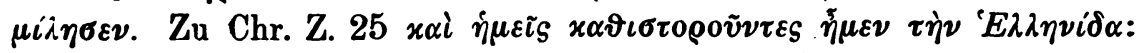

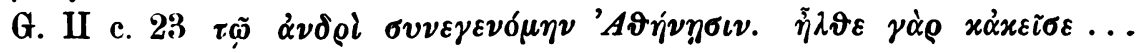




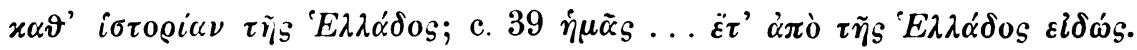

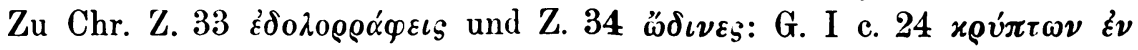

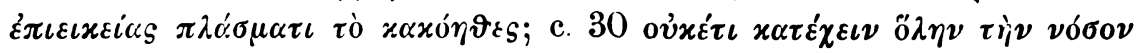

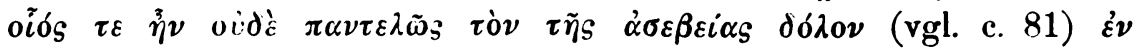

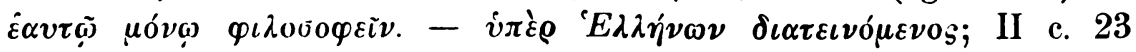

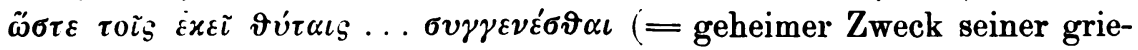

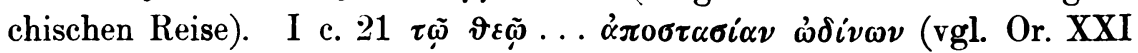

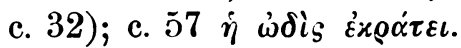

Diese biographische Partie ist mit einer Parallelkritik der christlichen und der hellenistischen Literatur durchsetzt, wie sie auch Gregor in der bereits erwähnten didaktischen Satire unternimmt. Außer den

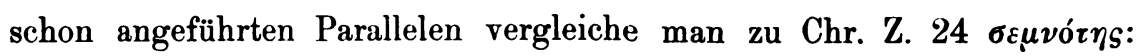

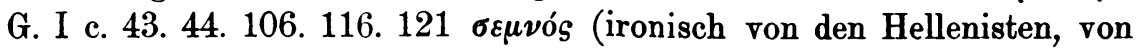

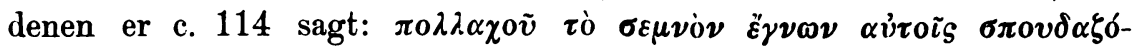

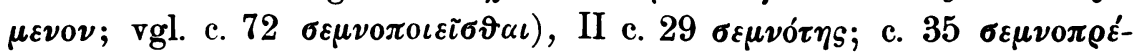

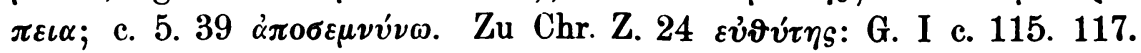
118. 119 das Verdikt über die hellenistischen $\alpha \lambda \lambda \eta \gamma o \rho \eta j \mu \alpha \tau \alpha . ~ Z u ~ C h r$.

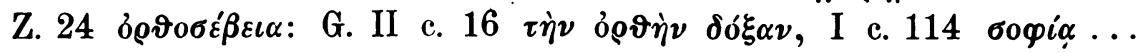

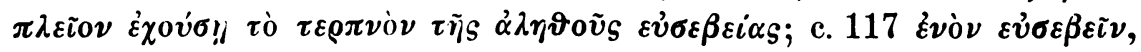

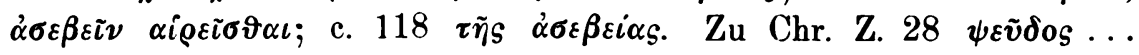
$\mu v \vartheta \iota x \delta$ : G. I c. $117 \tau \alpha \tilde{v} \tau \alpha \ldots \psi \varepsilon v \delta \tilde{\eta}$ (von dem auch G. I c. 113.117. 118. 119 , II c. 38 abgelehnten $\mu \tilde{v} \vartheta$ s). Zu Chr. Z. $29 \pi \lambda \dot{\alpha} \sigma \mu \alpha \tau \alpha$ : G. I c. 71 . 118. 124 , II c. $31.38 \pi \lambda \alpha^{\prime} \sigma \mu \alpha$ (vgl. II c. $\left.32 \pi \lambda \alpha^{\prime} \tau \tau \omega\right)$. Zu Chr. Z. 30. $31 \beta \varepsilon \dot{\beta} \eta \lambda o_{S}:$ G. I c. $86 \quad \beta \varepsilon \dot{\beta} \beta \lambda o_{S}$ (vgl. II c. $\left.29 \beta \varepsilon \beta \eta \lambda \delta \omega\right)$ ). Zu

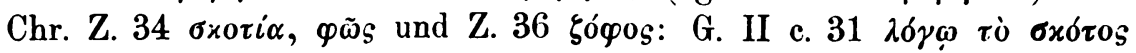

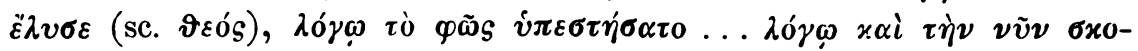

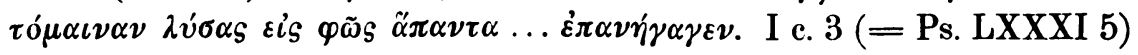

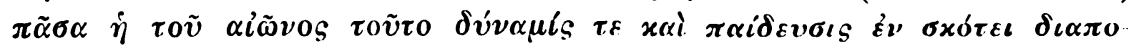

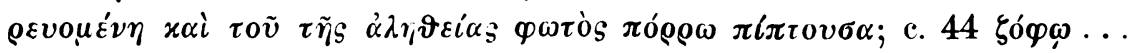

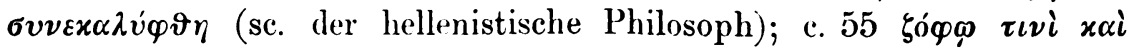

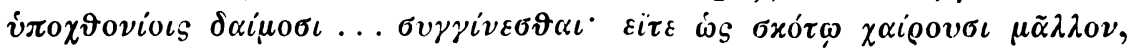

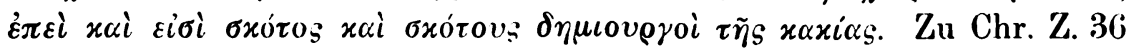

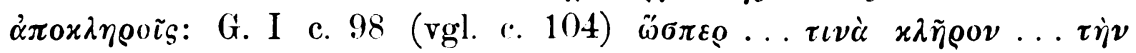

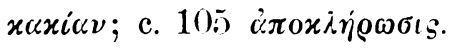

Trotz der entschiedenen Ablehnung, die Chrysoberges der hellenistischen Literatur zu teil werden läBt, gesteht er doch am Ende des dritten und am Anfang des vierten Abschnitts unter Hervorhebung ihres ästhetischen Reizes zu, daß er es zeitweilig mit ihrer ethischen Verwertung versucht habe. Auch Tregor spricht hiervon, jedoch, ia es ihm nur um die Beleuchtung der hellenistischen Lehrweise zu tun ist, lediglich rom Standpunkt dieser aus. Zu Chr. Z. 37 yó $\rho \gamma \alpha \lambda o v$ 


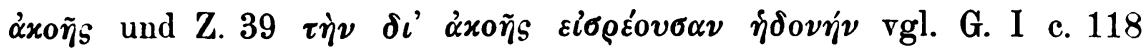

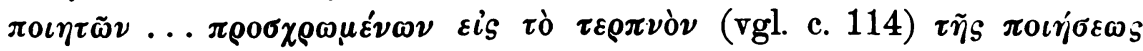

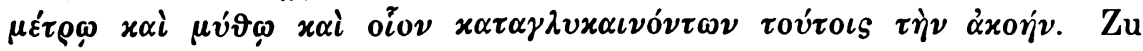

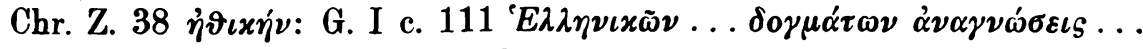

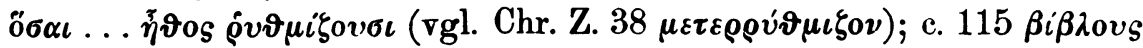

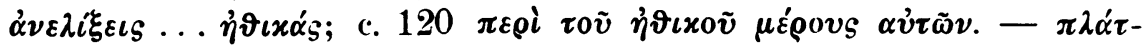

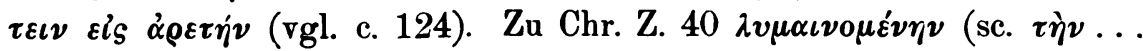

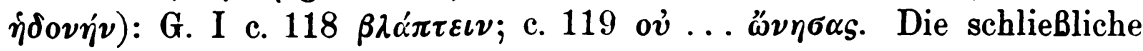
Verwerfung der hellenistischen Literatur Chr. Z. $44.47 \mathrm{ff}$. erinnert auch

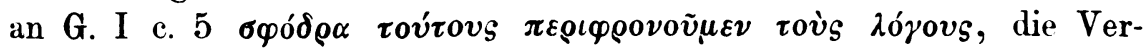

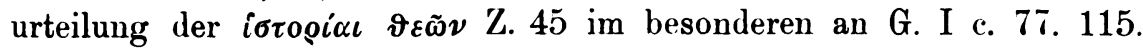

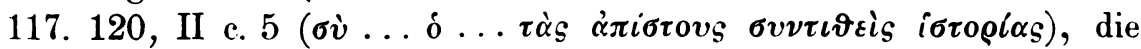
Kontrastrierung mit der christlichen $\pi \alpha i \delta \varepsilon v \sigma \iota s$ Z. 41 an G. I c. 105

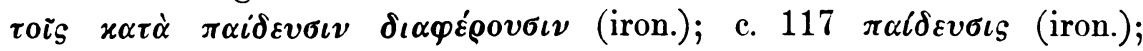

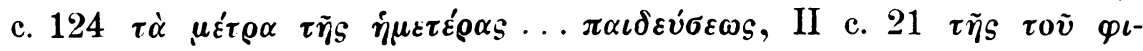

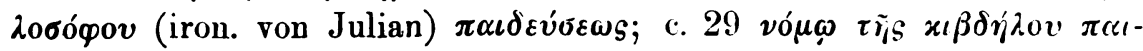
$\delta \varepsilon v ́ \sigma \varepsilon \omega \mathcal{Q} \alpha \dot{\alpha} 0 x \lambda \varepsilon i \sigma \alpha \nu \tau \varepsilon$ S. Vgl. I c. 73, II c. 1. 23. 34.

Im SchluB gemahnt der Vergleich der hellenistischen Literatur

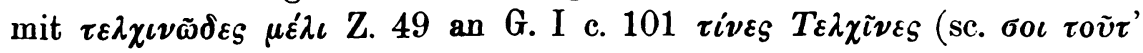

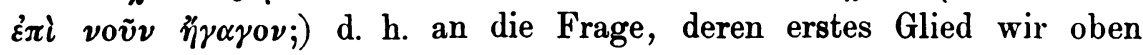
S. 130 mit dem Eingang der Ethopöie verglichen haben. Mit Chr.

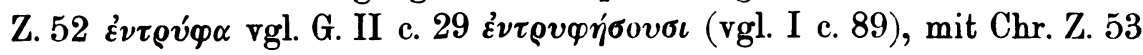

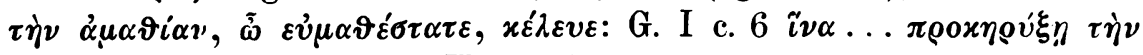

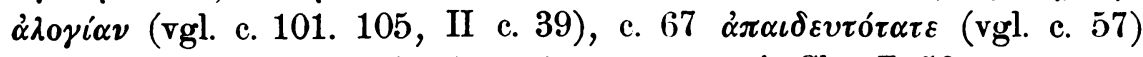

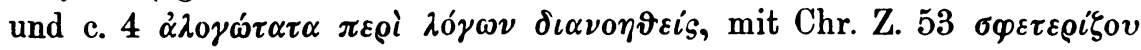
$x \tau \lambda$. außer dem bereits oben S. 131 angeführten Ediktfragment: G. I

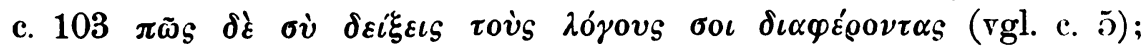

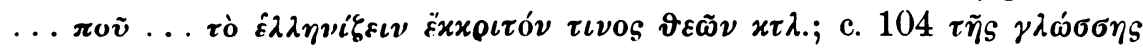

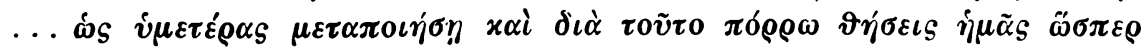

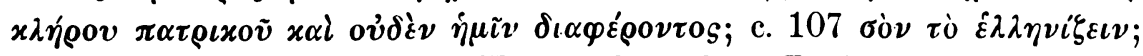

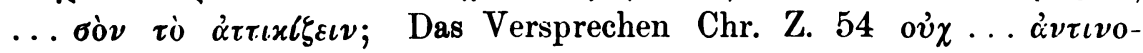
$\mu 0 \vartheta \varepsilon \tau \dot{\sigma} \sigma \omega$ klingt allgemein genommen an die Betonung der christlichen Feindesliebe G. II c. 36 und im besondern an G. I c. 77 und 98 an, wo einerseits der Verzicht der Christen auf das $\varphi \vartheta$ vovĩ (wie Chr. Z. 55) und $\alpha \dot{\nu} \tau \iota \pi \alpha i \xi \varepsilon \imath \nu$ hervorgehoben und andrerseits jede Analogie mit dem Rhetorenedikt auf christlicher Seite bestritten wird.

Die aufgezeigten Übereinstimmungen zwischen unserer Ethopöie und den Invektiven Gregors von Nazianz sind inhaltlich und formell so schlagend und in ihrer Fülle so ausgiebig, daB man an der unmittelbaren Abhängigkeit des Chrysoberges von dem Kappadokier nicht zweifeln kann. Vielleicht darf man für seine Bekanntschaft mit den 
Invektiven auch Or. I p. 9, 23 anführen, wo er Psalm XLIX 1 áxov́-

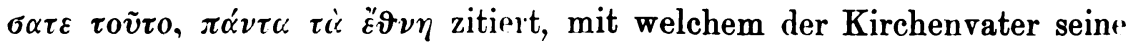
erste Streitrede gegen Julian beginnt. $\mathrm{DaB}$ er Or. I p. 6, 18 den auch von Gregor I c. 110 zum Vergleich beigezogenen Rhapsakes als Beispiel eines Tyramen nennt, erklärt sich, wie Treu a. a. 0. p. 44 zeigt, aus späteren Quellen. Aber das aus Gregor gewonnene Parallelenmaterial ist, namentlich für die positiver gehaltenen Partien der Ethopöie nicht erschöpfend. Da Gregor in dem Dedikationskapitel der zweiten Invektive (c. 39) diese Rede auch im Namen seines Gesinnungsgenossen und Mitstreiters Basilios überreicht und diesen hierdurch gewissermaßen zu seinem Mitarbeiter stempelt, so liegt es nahe, sich bei diesem nach weiterem Quellenmaterial umzusehen. Hierbei verfällt man angesichts

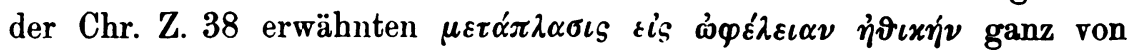

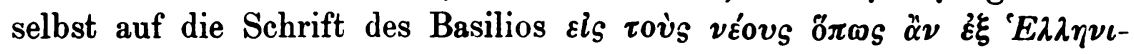

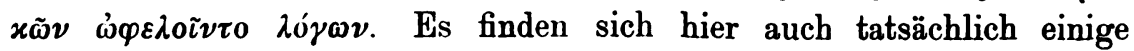

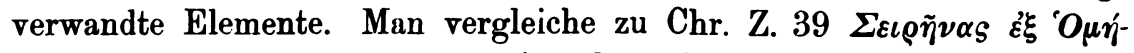

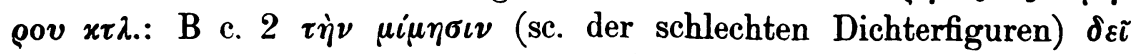

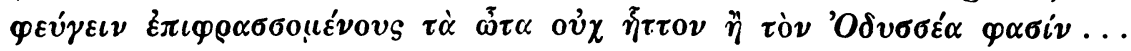
$\tau \dot{\alpha} \tau \tilde{\omega} \nu \Sigma \varepsilon \iota \rho \dot{\nu} \omega \nu \mu \varepsilon^{\prime} \lambda \eta$, zu Chr. Z. 45: B. c. 2 die Warnung $\pi \alpha^{\prime} \nu \tau \omega \nu$

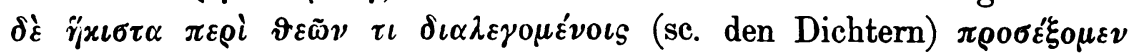

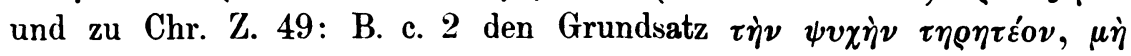

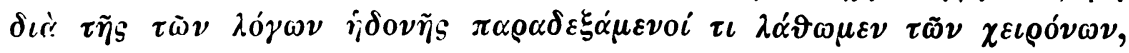

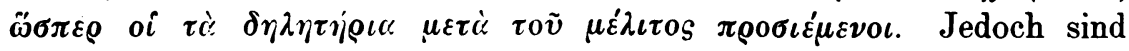
diese Anklänge - bei dem Fehlen des Kirkebeispiels - ebenfalls nicht erschöpfend und auch so sprichwörtlich gang und gäbe, dab sie nicht für beweisend gelten können. Ein Lehrer der Beredsamkeit konnte derartige Gemeinplätze, wie sie in den mythologischen Beispielen und den Z. 50 folgenden Vergleichen vorliegen, sehr wohl aus seinem im Lauf der Zeit aufgespeicherten eigenen Wissensschatze herausgreifen, ohne eine einzelne besondere Quelle ad hoc beizuziehen. Da wir oben S. 132 die biographischen Notizen der Ethopöie treffend aus Gregor belegen konnten, so ist hië für Chr. Z. 22 der Angabe des apokryphen

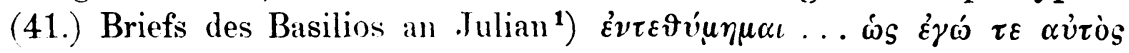

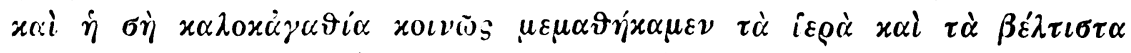

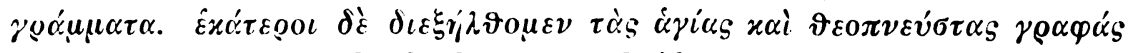
wohl mit Sicherheit als Quelle auszuscheiden.

Die Art, wie Chrysoberges die von uns nachgewiesene Quelle be-

1) Wir zitieren diesen Brief und die genannte Schrift des Basilius nach Bd. 4 (col. 345 B) und 3 (col.568 D sq.) des Migneschen Abdrucks der Garnierschen Ausgabe. 


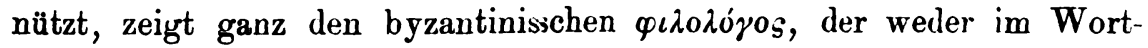
schatz noch in der Stilistik noch in den Gedanken irgendwelche Originalität besitzt. Wenn man es den Byzantinern als Hauptverdienst anrechuet, daß sie durch ihren Srammelfleiß uns die ihnen noch zugänglichen Reste der griechischen Liiteratur erhalten haben, so legt auch die Wahl des Themas unserer Etthopöie beredtes Zeugnis für diese konservative Tendenz ab. MuB main aber andrerseits auch die kommentierende Tätigkeit der Byzantiner: in Ehren halten, so hat Chrysoberges sich mit seinem $\pi \varrho \gamma^{\prime} \mu \nu \alpha \sigma \mu \alpha$ auch auf diesem Gebiete ein kleines Verdienst erworben. Hat er doch aus dem weitschichtigen Stoffe der Invektiven Gregors von Nazianz: den urkundlich interessantesten Kern herausgeschält, paraphrasiert umd eben dadurch seinen Schülern und Lesern rerständlicher gemacht. $\mathrm{DaB}$ er sein Thema überhaupt bei dem Nazianzener finden konnte, beweist für diesen, wie nahe er selbst schon dem Byzantinismus stehit. Auf jeden Fall aber haben wir in dem Wiederaufgreifen desselbem einen unverächtlichen Beleg für die historische Kontinuität des Glaubens an den Bildungswert des griechischen Schrifttums zu erblicken. Daß sich diese Anerkennung bei Chry-

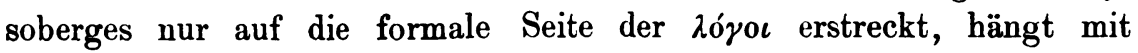

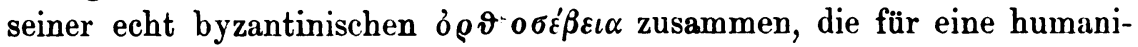
stische Auffassung derselben noch nicht reif war.

$$
\text { Freiburg i. B. }
$$

J. R. Asmus. 